\title{
Alpha Hypersynchrony and Atypical Memory Processes in Soldiers with Post-Traumatic Stress Disorder
}

\author{
Benjamin T. Dunkley ${ }^{1,2,3^{*}}$, Elizabeth W. Pang ${ }^{2,4}$, Paul A. Sedge ${ }^{5}$, Rakesh Jetly ${ }^{5}$ and Margot J. Taylor ${ }^{1,2,3,6}$ \\ ${ }^{1}$ Department of Diagnostic Imaging, The Hospital for Sick Children, Toronto, Canada \\ ${ }^{2}$ Neuroscience \& Mental Health Program, The Hospital for Sick Children Research Institute, Toronto, Canada \\ ${ }^{3}$ Department of Medical Imaging, University of Toronto, Toronto, Canada \\ ${ }^{4}$ Division of Neurology, The Hospital for Sick Children, Toronto, Canada \\ ${ }^{5}$ Directorate of Mental Health, Canadian Forces Health Services, Ottawa, Canada \\ ${ }^{6}$ Department of Psychology, University of Toronto, Toronto, Canada
}

\section{Correspondence to:}

Benjamin T. Dunkley, PhD

Department of Diagnostic Imaging.

555 University Ave, Toronto

Canada, M5G 1X8, Canada

Tel: (416)-813-7654 ex 309117

E-mail: ben.dunkley@sickkids.ca

Received: March 21, 2016

Accepted: June 06, 2016

Published: June 13, 2016

Citation: Dunkley BT, Pang EW, Sedge PA, Jetly R, Taylor MJ. 2016. Alpha Hypersynchrony and Atypical Memory Processes in Soldiers with Post-Traumatic Stress Disorder. I Neuroimaging Psychiatry Neurol 1(2): 54-63.

Copyright: () 2016 Dunkley et al. This is an Open Access article distributed under the terms of the Creative Commons Attribution 4.0 International License (CC-BY) (http://creativecommons. org/licenses/by/4.0/) which permits commercial use, including reproduction, adaptation, and distribution of the article provided the original author and source are credited.

Published by United Scientific Group

\begin{abstract}
Post-traumatic stress disorder (PTSD) is a psychiatric disorder that is associated with a variety of cognitive sequelae, including disorders of memory. We hypothesised that alterations in functional connectivity in PTSD, mediated by neural synchronisation, may be associated with such deficits. Using magnetoencephalography (MEG), we identified elevated alpha-band $(8-14 \mathrm{~Hz})$ phase synchronisation in soldiers with PTSD during working memory and delayed recognition tasks that included war-related and neutral pictures. Performance for the PTSD group matched controls on the working memory task, and this was accompanied by increases in long-range connectivity in the alpha-band during encoding, left-dominant, at 50-150 ms and during recognition at $150-250 \mathrm{~ms}$ involving temporal and frontal brain regions. In the delayed recognition task, performance was reduced in the PTSD group compared to controls for neutral, but not war-related images. Elevated alpha-band phase synchronisation was observed in the PTSD group in a right-dominant network of front-temporal and deep grey matter regions for both types of stimuli between 50-150 ms. The literature posits alpha phase dynamics are involved in working memory; our results suggest that atypical alpha synchronisation is related to memory-related sequelae in PTSD, one of the more ubiquitous cognitive comorbidities observed in this disorder.
\end{abstract}

\section{Keywords}

Post-traumatic stress disorder, Memory, Magnetoencephalography (MEG), Functional connectivity, Military/combat

\section{Introduction}

Post-traumatic stress disorder (PTSD) is a psychiatric condition which develops after experiencing a disturbing life event. PTSD is defined as comprising four symptom clusters that encompass re-experiencing, avoidance behaviours, emotional numbing and hyper-vigilance, and negative cognitions and moods $(\mathrm{DSM}-\mathrm{V})$ [1]. Approximately 50\% of the general population experience at least one traumatic event during their lifetime, with the incidence of the disorder at around $5-10 \%$ [2]. However, in military populations the prevalence of PTSD is found to be higher, especially in those returning from combat deployment [3].

In PTSD, studies report deficits in a number of cognitive domains [48], including short-term working memory (WM) $[9,10]$ and delayed recall 
[10-12]. Memory processing difficulties are significant as autobiographical and episodic memories are thought to be the principal component of the re-experiencing symptoms for those with PTSD. Critical for recall and re-experiencing of memories are the medial temporal lobes, and the hippocampal structures in particular [13]. These temporal lobe structures are crucial for the perceptual richness of memories; i.e., the vivid evocation of both the mental and perceptual components associated with an event in one's life [14-17].

It has been suggested that the ability to recollect memories with general affective, and especially negative, content is comparatively increased in PTSD [18]; conversely, information regarding precise occurrences and context is compromised [18]. Generally, this implies that specific memory-related cognitive resources are prioritised to process threat-related and/or affective information, and neutral information is relegated to low-priority processing, incurring better performance for the former and reduced performance for the latter [19].

The emotional aspect of episodic memory would predict that emotion processing regions are implicated in memoryrelated dysfunction in PTSD [20]. fMRI studies of WM have revealed increased activity in those with PTSD in emotional processing networks including the amygdala, ventrolateral and medial prefrontal cortex, and concomitant decreases in dorsal executive function networks [21-23]. The interactions of these regions during memory tasks have been less investigated.

Interactions among brain areas mediated by oscillatory synchronisation are thought to underlie the exchange and temporal coordination of information required for cognition [24-26]. Numerous studies have shown that frequencyspecific interactions across the cortex play a critical role in memory. Magnetoencephalography (MEG) has shown this to be the case in visual working memory processing, with evidence that alpha synchronisation performs a critical role in WM, with both large-scale, global $[27,28]$ and small-scale, local interactions $[29,30]$ at this frequency scale contributing to the maintenance and manipulation of stored information.

Inter-regional synchrony has been informative in understanding the cortical pathophysiology of PTSD [31,32] with alterations to slow-wave amplitudes noted in left temporal, right frontal, and right parietal cortex [33, 34]. Recently, we have shown that high-frequency synchronisation during rest distinguishes soldiers with PTSD from control soldiers [35]. These studies suggest abnormal synchrony among brain regions might underlie some of the cognitive sequelae of the disorder. However, knowledge remains scant regarding taskdependent changes in connectivity in PTSD populations. We investigated the role of oscillatory synchronisation in working memory and delayed recognition tasks in soldiers with PTSD using MEG. We examined task-dependent, frequency-specific interactions in neuronal networks using network statistics and graph theoretical analysis. We hypothesised that our PTSD group would show decreased memory function, and that this would be accompanied by reduced functional connectivity mediated by oscillatory synchronisation, a mechanism thought to support normative memory function.

\section{Methods and Materials}

\section{Participants}

MEG data were recorded from 48 Canadian Armed Forces soldiers, who were deployed in frontline roles in support of the Afghan mission. Twenty-four soldiers diagnosed with PTSD (all male, mean age $=37.67 \pm 1.39$ years) and 24 combat-exposed soldiers without PTSD (all male, mean age $=$ $33.97 \pm 0.98$ years) were recruited. All participants underwent cognitive-behavioural testing and completed a number of tasks in the scanner during the study; all had normal or correctedto-normal visual acuity. All participants gave informed written consent. Participants were initially approached by a military clinician to ask if they wished to participate in the study. If they were interested, their names were then passed to a research assistant, who established contact to confirm willingness to participate in the study. All procedures were approved by the Hospital for Sick Children and Canadian Armed Forces Research Ethics Boards.

Inclusion criteria for the PTSD group were: a clinical diagnosis of PTSD at an operational trauma stress support centre (OTSSC) as determined by a psychiatrist or psychologist specializing in trauma-related psychiatric disorders; PTSD symptoms present between 1 and 4 years prior to taking part in the study; regular mental health follow-ups; and current PTSD check list (PCL) scores of $>50$, indicating the presence of moderate to severe PTSD.

The diagnosis was determined through a comprehensive, semi-structured interview with a clinician based upon DSM-IV-TR diagnostic criteria [36], along with Canadian Armed Forces (CAF) standardized psychometric testing. All participants in the PTSD group were recruited from one of the CAF OTSSCs. There were usually more than one DSMIV-TR 'A1' stressor-related criteria (direct personal experience of an event that involves actual or threatened death or injury) [36], identified as a traumatic event contributing to the development of PTSD, with diagnosis related to operational exposure. Control soldiers were combat-exposed, frontline troops in similar military roles, and selected from cohorts of comparable rank, education level, and military experience. Additional inclusion criteria were applied to both groups: no history of a traumatic brain injury (TBI), as screened by a psychiatrist through a review of their electronic health record, telephone interview, and administration of the Defense and Veteran's Brain Injury Centre (DVBIC) screening tool.

Exclusion criteria included ferrous metal inside the body or implanted medical devices that would be MRI contraindications or interfere with MEG data acquisition; seizures or other neurological disorders; certain ongoing medications (anticonvulsants, and/or benzodiazepines, or other GABA antagonists) known to directly or significantly influence EEG findings. This was a naturalistic study and PTSD participants were undergoing treatment including evidenced-based psychotropic medication(s), such as selective serotonin reuptake inhibitors (SSRIs), serotoninnorepinephrine reuptake inhibitors (SNRIs), and Prazosin. Whilst there is potential for these drugs to be a confounding factor given their psychoactive nature, it was deemed unethical 
to request withdrawal from treatment due to their long halflife and the associated risk of suicide from halting intervention for extended periods.

\section{Cognitive-behavioural evaluation}

All soldiers completed short cognitive-behavioural assessments at the time of the MEG studies, including the Wechsler Abbreviated Scale of Intelligence (WASI), the Generalized Anxiety Disorder 7-item Scale (GAD7), the Patient Health Questionnaire (PHQ9) for depression, the Conners ADHD scale, $3^{\text {rd }}$ ed. to identify attention disorders, and for those with PTSD, the Post Traumatic Stress Disorder Check List (PCL). Means, standard deviations and inferential statistics for the groups are in Table 1.

Table 1: Cognitive-behavioural and clinical assessment measures for PTSD and control soldiers.

\begin{tabular}{|c|c|c|c|}
\hline & PTSD & Control & Test statistic \\
\hline$n$ & 24 & 24 & \\
\hline Conners & $24.50(9.58)$ & $6.50(4.91)$ & $t(46)=8.19, p<0.001$ \\
\hline GAD-7 & $15.13(4.33)$ & $1.92(1.87)$ & $t(46)=13.74, p<0.001$ \\
\hline PHQ9 & $16.88(4.99)$ & $1.92(2.24)$ & $t(46)=13.41, p<0.001$ \\
\hline PCL & $64.09(7.67)$ & N/A & N/A \\
\hline
\end{tabular}

Conners: Attention-Deficit Hyperactivity Disorder Test

GAD-7: Generalized Anxiety Disorder 7

PHQ9: Patient Health Questionnaire

PCL: Post Traumatic Stress Disorder Check List

\section{Procedure}

\section{Task 1: working memory}

Participants completed an N-back memory task (Figure 1 , top) adapted for MEG. They viewed a series of stimuli consisting of 110 war-related (combat-related, emotionallysalient) scenes and 95 neutral scenes matched on the initial scene similarity. Termed the '1-back' task, each war-related image was shown only once during the run and not repeated; randomised immediate repeats of a subset of the neutral images were shown after the initial presentation. Participants were instructed to hold each image in memory and respond as fast as possible to the repeated presentation of stimuli using an MEG-compatible button-box.

245 trials were presented in total (110 combatrelated war-related images, 95 neutral 'new' images, and 40 neutral 'repeated' images) using Presentation software (Neurobehavioral Systems, Inc., Berkeley CA) via a back projection screen placed $78 \mathrm{~cm}$ from the participants' eyes. Stimuli subtended a visual angle of $21^{\circ}$ horizontally by $13^{\circ}$ vertically and lasted for $200 \mathrm{~ms}$. In between each presentation a centrally-presented fixation cross was shown, with a jittered presentation time of 1050-1300 ms (inter-stimulus interval).

\section{Task 2: delayed recognition}

In the delayed recognition task about 40 minutes later, 80 images from the previous experiment were shown, 40 neutral and 40 war-related images, interspersed with 40 foils (20 new war-related and 20 new neutral; Figure 1, middle). Participants had to recognise whether the image had been presented in the earlier experiment, and respond with either 'new' (not seen previously) or 'old' (previously seen). As before, stimuli subtended a visual angle of $21^{\circ}$ horizontally by $13^{\circ}$ vertically and were presented until a response was recorded.

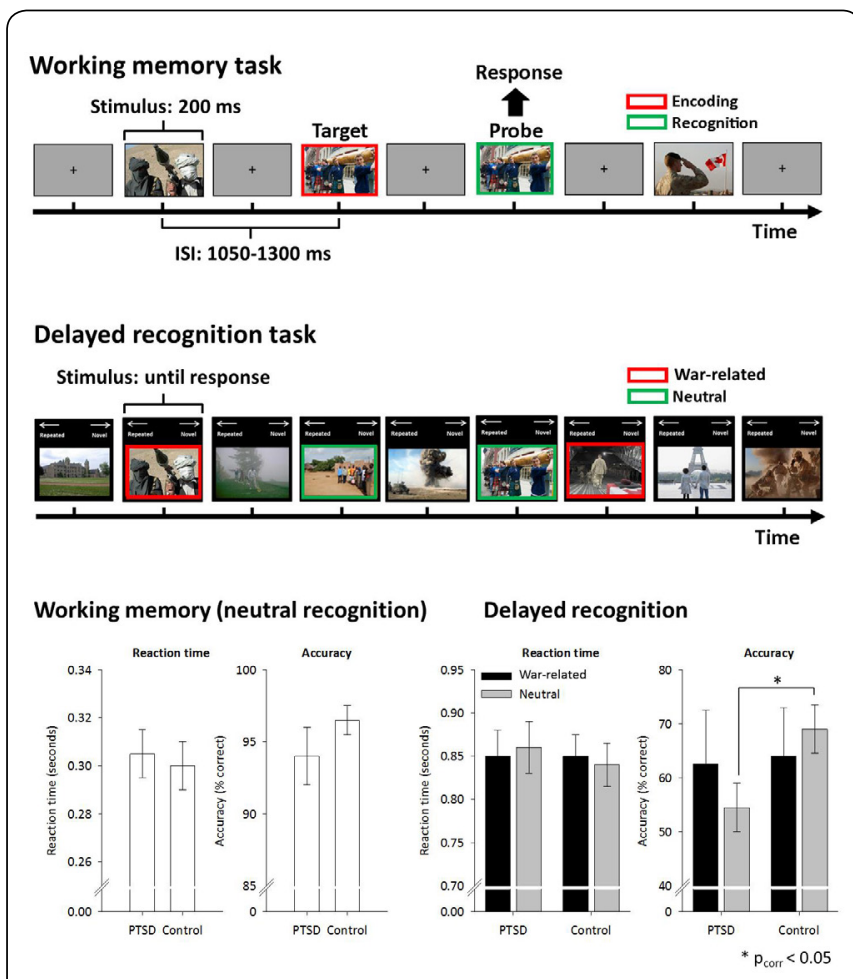

Figure 1: Experimental schematic and behavioural results for the working memory (1-back) and delayed recognition tasks. Top; Warrelated images are shown once and a randomised subset of neutral images are shown twice in succession (1-back probe/encoding image, and target/recognition image subsequently displayed). Images were pseudo-randomised in terms of presentation order. Participants had to respond as quickly as possible when they recognised the 1-back target image. Middle; 80 images from the previous experiment were shown again, 40 neutral and 40 war-related images, interspersed with 40 foils (20 war-related and 20 neutral). Participants had to respond to each image with either 'Novel' or 'Repeated'. Behavioural results from the working memory and delayed recognition task for the PTSD and control soldiers. Bottom left; Mean performance measured by reaction time and accuracy (\% correct trials) was the same when comparing between the groups for the recognition trials in the 1 -back task $( \pm 1$ standard error bars). Bottom right; No significant differences were found between the groups in the war-related image trials for the delayed recognition task, although the PTSD group were significantly less accurate in correctly distinguishing the neutral images ( ${ }^{*}$ Bonferonni $\left.\mathrm{p}_{\text {corr }}<0.05\right)$ compared to the control soldiers.

\section{Imaging data acquisition}

MEG data were collected inside a magnetically-shielded room on a CTF Omega 151 channel system (CTF Systems, Inc., Coquitlam, Canada) at the Hospital for Sick Children. Data were recorded at $600 \mathrm{~Hz}$ sampling rate with third-order spatial gradient environmental noise-cancellation. Throughout the run, head position was continuously recorded by three fiducial coils placed on the nasion, and left and right preauricular points.

After the MEG session, anatomical 3T MRI images were acquired (Magnetom Tim Trio, Siemens AG, Erlangen, Germany), T1-weighted magnetic resonance images using 
high-resolution 3D MPRAGE sequences on a 12 channel head coil. MEG data were co-registered to each individual's MRI structural image using the reference fiducial coil placements.

\section{MEG Data Processing}

\section{Seed definition and virtual electrode analyses}

MEG data were band-pass filtered offline at $1-150 \mathrm{~Hz}$, a notch filter applied at $60 \mathrm{~Hz}(8 \mathrm{~Hz}$ bandwidth). Sensorlevel time series data were visually inspected and trials with significant artefacts related to head-motion were removed from subsequent analysis; this was supplemented by headmovement recordings to confirm visual assessments.

The Automated Anatomical Labeling (AAL) atlas [37] was used to identify 90 sources (seeds) in cortical and subcortical regions. These coordinates defined locations for time-series to be extracted and analyzed (see Figure S1 and Table S1 of Supplementary materials). Broadband time-series ('virtual electrodes') from these voxels were reconstructed using a vector beamformer on the basis of the $90 \mathrm{AAL}$ coordinates for each subject and filtered into five classical EEG bandwidths for further analyses: theta (4-7 Hz), alpha (8-14 Hz), beta $(15-30 \mathrm{~Hz})$, 'low' gamma $(30-80 \mathrm{~Hz})$, and 'high' gamma (80-150 Hz).

Beamformers are a type of spatial filter used to optimize sensitivity to activity in a given brain location of interest (such as one of the 90 seed regions), whilst attenuating signals from other locations. Individual weight vectors are applied to each sensor measurement and summated to give an estimated source activity to a particular cortical seed location [38]. Additionally, MEG beamformers are effective at suppressing ocular artefacts generated by eye movements (and in particular, blinks), as well as other non-ocular physiological artefacts, such as cardiac and muscle activity [39].

\section{Assessing functional connectivity: Phase lag index}

Each of the 5 band-pass filtered data sets was then submitted to a functional connectivity analysis, using the phase lag index (PLI) [40]. The instantaneous phase of each sample from the filtered time-series was calculated using the Hilbert transform. The cross-trial degree of phase synchronisation for every time point between all pair-wise combinations of the seeds was computed using the PLI, which is based on the magnitude of the imaginary component of the cross-spectrum [40]. Ranging between 0 and 1 , these values quantify the phase synchrony between two sources, referred to as functional connectivity.

\section{Statistical analyses}

Adjacency matrices with PLI values acting as edge weights for all sources were constructed at every time point/ sample, which resulted in a 90x90 [x5 frequencies $\mathrm{x} 720$ samples/time points] matrix of weighted undirected graphs for each participant. For the generation of statisticallythresholded functional connectivity images, temporallyaveraged adjacency matrices over time windows of interest were generated, and statistical analyses were performed on the resulting matrices using the Network Based Statistic (NBS)
[41]. NBS first applies an initial univariate threshold to each analyzed edge. The extent of connectivity components, defined as contiguous groups of nodes connected by suprathreshold connections, is then obtained. Group membership is then shuffled and the extent of the largest component which occurs in this surrogated data is recorded, and this process is repeated 5000 times to generate a null distribution. The ranking of connectivity components from the unshuffled data in the surrogate distribution is used to determine statistical confidence; as the surrogate distribution considers the largest connectivity component that could occur, assuming the null hypothesis across the entire analyzed network, this approach is very effective in controlling for false positives due to multiple comparisons at any threshold.

In the present analysis, the initial univariate threshold was set at a moderate t-value of $3[41,42]$. Brain networks were visualized using BrainNet Viewer [43]. Further analyses, such as permutation testing of between-group differences of adjacency matrices, were completed using scripts written inhouse.

\section{Results}

\section{Cognitive-behavioural assessment}

Cognitive-behavioural assessment measures for PTSD and Control soldiers are shown in Table 1. Consistent with their diagnosis, soldiers with PTSD showed increased incidence of attentional problems, anxiety, and depressive symptoms (t-tests, all $\mathrm{p}<0.001$ ). The soldiers with PTSD also had a slightly lower IQs; it has been shown that IQ might be a predictor of susceptibility to PTSD [10], and is certainly associated with the presence of PTSD (i.e., performance is impacted by PTSD), as are other psychiatric disturbances.

\section{Task performance}

Figure 1, bottom left, highlights the behavioural results from the immediate recognition aspect of the working memory task; performance was comparable between the groups (measured as a function of reaction time and task accuracy), with no significant difference observed in either the RT or accuracy metrics ( $\mathrm{t}$-test, $\mathrm{p}>0.05$ ). In the delayed recognition task (Figure 1, bottom right), a mixed-effects ANOVA was used to compare within (stimulus type - war vs. neutral) and between-subjects (group - PTSD vs. control) factors. There was no significant effect on reaction time of stimulus type, $\mathrm{F}(1,46)=1.24, \mathrm{p}=0.271$, of group, $\mathrm{F}(1,46)=0.25$, $\mathrm{p}$ $=0.619$, or any interaction effect, $\mathrm{F}(1,46)=0.08, \mathrm{p}=0.786$. An ANOVA on accuracy revealed a non-significant effect of stimulus type, $\mathrm{F}(1,46)=0.81, \mathrm{p}=0.373$, of group, $\mathrm{F}(1,46)=$ $1.81, \mathrm{p}=0.185$; however, there was a significant condition $\mathrm{x}$ group interaction, $\mathrm{F}(1,46)=11.11, \mathrm{p}=0.002$. Post-hoc tests contrasting between-group accuracy for individual condition levels (stimulus type, either by war or neutral pictures) revealed a non-significant difference between the PTSD group and control soldiers for war-related stimuli (black bars; $\mathrm{t}(46)=$ $0.28, \mathrm{p}=0.784)$. For the neutral stimuli, however, the PTSD group were significantly less accurate compared to controls (grey bars; $(46)=2.376, \mathrm{p}_{\text {corr }}=0.022$ ). 


\section{MEG connectivity: working memory}

Figure 2 shows group-mean, whole-brain connectivity strength time-series in the alpha band during encoding the first presentation of the stimuli that were repeated (i.e., encoding) - (top left panel) and recognition (top right panel) trials in the working memory 1-back task. Time-series plots in the other 4 frequency bands showed minor differences between the groups in this task (see Figure S2 of Supplementary materials); these were submitted to an exploratory NBS analysis, but no significant differences were found.

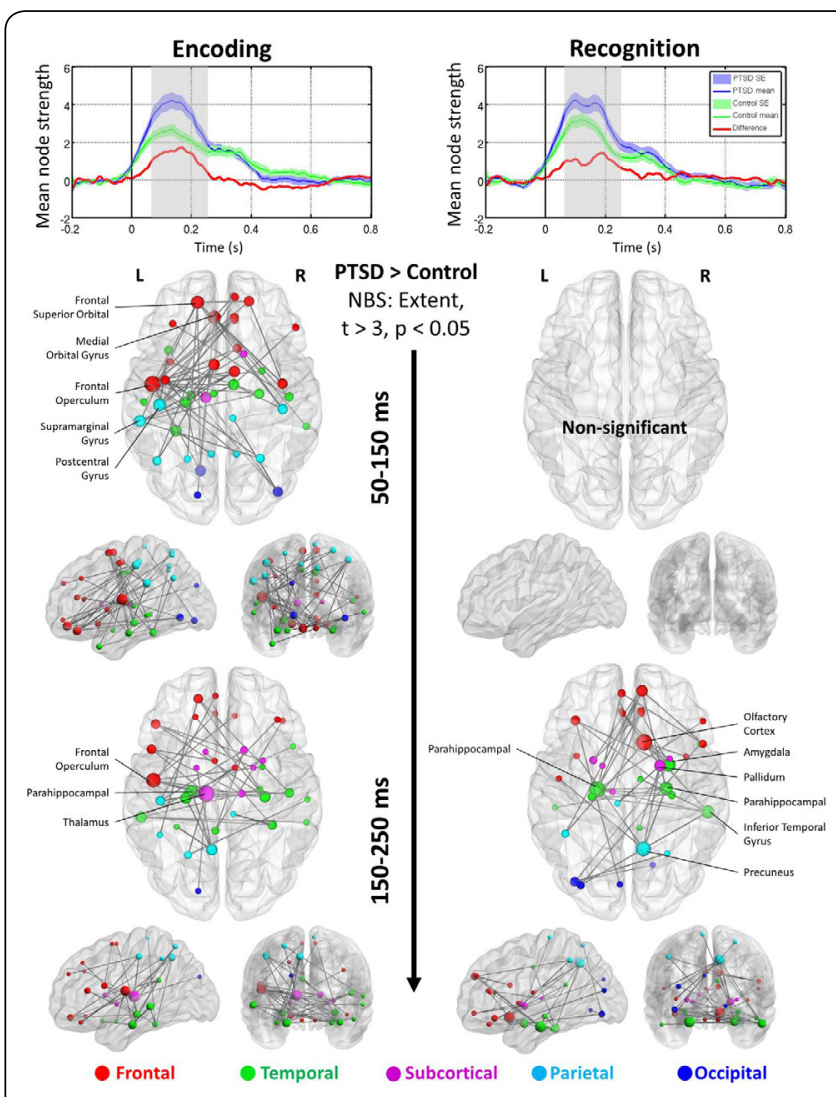

Figure 2: Group-mean, whole-brain connectivity strength in the alpha band during an average encoding and recognition trial (-0.2 to 0.8 seconds, stimulus appears at 0 time point; \pm 1 standard error bars). The PTSD group (blue trace) show large increases in alpha band connectivity strength across the network compared to control soldiers (green trace; difference between the groups in red) in both the encoding (top left panel) and recognition (top right panel) trials. Group contrasts in connectivity were constructed for two non-overlapping windows at the $50-150$ and $150-250 \mathrm{~ms}$ time points (denoted by shaded area). NBS comparisons revealed large-scale elevated alpha synchronisation in frontal and temporal regions for both time windows in the encoding trials. No significant difference was found between the groups in recognition trials for the $50-150 \mathrm{~ms}$ window, but significant connectivity differences were found in the 150-250 time window (NBS: Extent method, $\mathrm{t}>3, \mathrm{p}<0.05$; node radius is scaled by degree difference, or how many significant connections link a node to the network). Labels nodes are significant regions with degree $>4$.

The PTSD group (blue trace) displayed task-dependent increases in the mean alpha-band node strength (the sum of the connection weights between that node and the rest of the network) compared to control soldiers (green trace; difference between the groups in red) in both the encoding and recognition aspects of the task. Both groups appear to peak in connectivity at approximately $100-200 \mathrm{~ms}$ post-stimulus presentation; therefore, group differences in connectivity were examined around this time window to capture the temporal manifestation and decline in connectivity changes as a result of memory processing. In particular, we wanted to capture increases in connectivity towards the peak response (50-150 $\mathrm{ms})$, and decreases moving on from the peak (150-250 ms).

Mean connectivity adjacency matrices were generated for two non-overlapping windows defining the 50-150 and 150-250 ms time points for each subject, concatenated into 3-dimensional matrices separated by group (PTSD and control), and then submitted to an independent NBS analysis. NBS comparisons revealed elevated, large-scale alpha synchronisation in frontal and temporal regions for both time windows in the encoding aspect of the task (NBS: Extent method, $\left.\mathrm{t}>3, \mathrm{p}_{\text {corr }}<0.05\right)$. No significant difference was found between the groups in recognition trials for the $50-150 \mathrm{~ms}$ windows, but significant connectivity differences were found in the 150-250 ms time window. Similar to the encoding aspects of the task, these network differences in connectivity in the 150-250 ms window appeared prominently between temporal and frontal seed regions for correct recognition (see Table S2 \& S3 in Supplementary materials).

Differences in whole-brain network interactions were then assessed by defining lobe-lobe communication using regional-mean adjacency matrices (Figure 3). These were obtained by averaging PLI values across all inter-regional connections for a given comparison (across all connections within the right frontal cortex, or across all connections between right temporal and left parietal seed locations, for

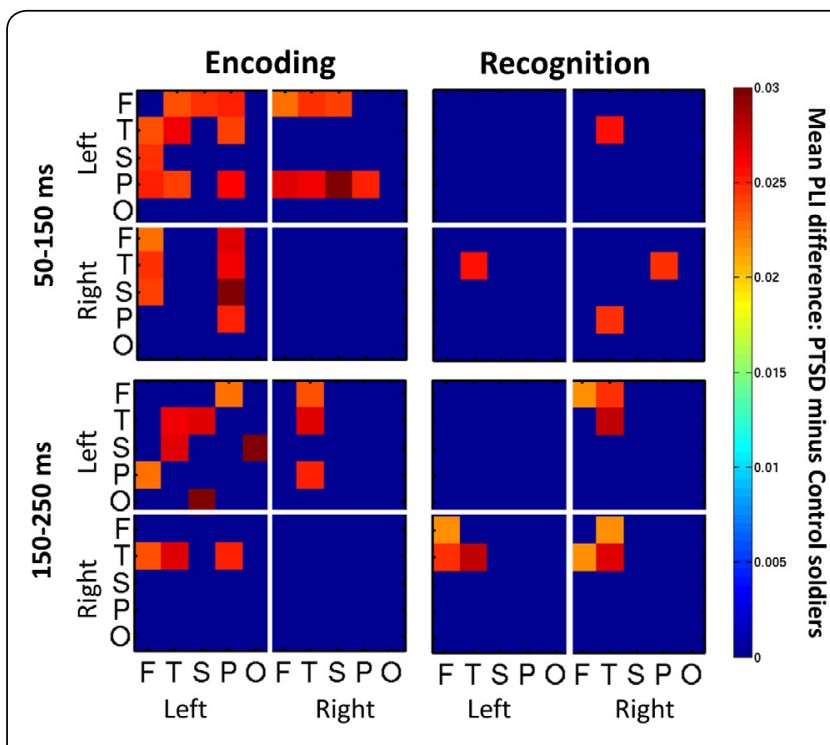

Figure 3: Adjacency matrices depicting between-groups differences (PTSD minus control group) in lobe-lobe interactions by hemisphere, for the encoding (left panels) and recognition trials (right panels) in the $50-150 \mathrm{~ms}$ (top panels) and $150-250 \mathrm{~ms}$ (bottom panels) time windows (dark blue elements indicate $\mathrm{p}>0.05$, warm coloured elements indicate significant differences from permutation testing $\mathrm{p}<0.05)$. For neutral encoding, PTSD soldiers show elevated lobe-lobe interactions in the 50-150 and $150-250 \mathrm{~ms}$ time windows compared to controls, and these elevated dynamics are especially prominent for intrahemispheric interactions in the left hemisphere (top left quadrant). F: frontal, T: temporal, S: sub-cortical, P: parietal, O: occipital. 
example). Here, between-groups alterations in connectivity (PTSD minus control group) for lobe-lobe interactions by hemisphere were examined for encoding and recognition for the baseline-corrected 50-150 ms and 150-250 ms windows. PTSD soldiers showed elevated lobe-lobe interactions for the encoding aspect of the task in the 50-150 ms and 150-250 ms time windows compared to controls, which were especially prominent in the left hemisphere (top left quadrant of Figure 3; significant differences after permutation testing, $\mathrm{p}_{\text {corr }}<0.05$ ). These differences were less pronounced in the $150-250 \mathrm{~ms}$ window. In the recognition aspects of working memory, PTSD soldiers showed increased connectivity that was especially apparent in the $150-250 \mathrm{~ms}$ window, in bilateral frontotemporal networks, with a bias for right intra-hemispheric fronto-temporal interactions (see Table S4 in Supplementary materials).

\section{MEG connectivity: delayed recognition}

As with the 1-back task, the time-series plots showed significant differences in the alpha band, and little differential connectivity in the other 4 frequency bands (see Figure S3 in Supplementary materials). Figure 4 shows group-mean, whole-brain connectivity strength time-series in the alpha band for war-related (top left panel) and neutral (top right panel) trials in the delayed recognition task. Increased alpha band connectivity strength was observed in the PTSD group compared to control soldiers in response to both types of stimuli (top right panel). Similar to the working memory task, two non-overlapping time-windows were chosen to explore group differences in connectivity around the peak response, the 50-150 ms and 150-250 ms time-windows.

NBS comparisons revealed significantly increased alpha synchronisation for both war-related and neutral trials in the PTSD group in the 50-150 ms time window (NBS: Extent method, $\mathrm{t}>3, \mathrm{p}_{\text {corr }}<0.05$ ); no significant differences in connectivity patterns were observed in the 150-250 ms window. For war-related stimuli, frontal and temporal regions appeared hyper-synchronised and significantly overconnected (high-degree) in frontal and temporal regions. Strong connectivity was also seen in nodes in left occipital cortex, consistent with the saliency of the visual imagery (see Table S5 in Supplementary materials).

Similar patterns of hyper-connectivity were noted in the neutral trials for the PTSD group compared to controls, although the spatial extent of network differences appeared diminished compared to the war-related stimuli; however, there were still a number of high-degree nodes present within this network (see Table S6 in Supplementary materials).

Adjacency matrices depicting between-group differences (PTSD minus control group) in lobe-lobe interactions by hemisphere in the 50-150 ms window are shown in Figure 4, bottom panels (significant differences after permutation testing, $\left.\mathrm{p}_{\text {corr }}<0.05\right)$. War-related stimuli induced elevated interactions within the right hemisphere for the PTSD group, with frontotemporal, temporo-subcortical and within-subcortical sources exhibiting hyper-connectivity; this pattern was absent for neutral trials. This suggests that while large-scale connectivity during correct recognition of war-related and neutral stimuli appear similar initially, when connectivity is examined at the regional level, it is quantitatively different when probing interactions at the regional level (when we compare the adjacency matrices in Figure 4).

To examine whether individual variability in performance for neutral trials on the delayed recognition task was related to alpha synchrony, we calculated correlations of connectivity during the 50-150 ms time window versus performance. Correlations of mean, whole-brain connectivity versus accuracy analyses revealed significant correlations for both the PTSD $(r=0.49, p=0.023)$ and control groups $(r=0.43, p=$ 0.036).

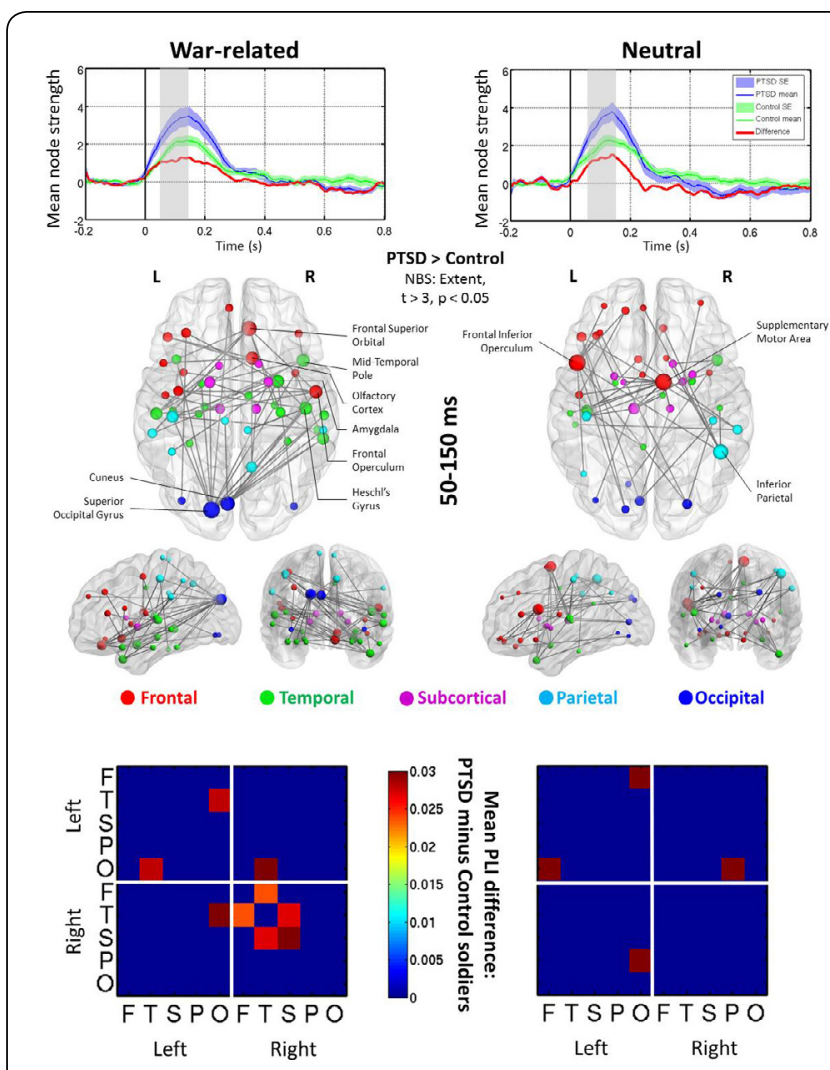

Figure 4: Group-mean, whole-brain connectivity strength in the alpha band during correct trials for the average war-related and neutral trials $(-0.2$ to 0.8 seconds, stimulus appears at 0 time point, \pm 1 standard error bars). The PTSD group show increased alpha band connectivity strength, with significant connectivity differences between the groups for both trial types in the 50-150 ms window (NBS: Extent method, $t$ $>3, \mathrm{p}<0.05)$. Network alterations for war-related trials appear across diffuse, large-scale networks, particularly in bilateral frontal, subcortical and temporal regions; there are also high-degree, hyperconnected nodes in left occipital cortex. Elevated connectivity in the neutral trials was also observed. No significant differences were observed in the 150-250 ms time window $(p>0.05)$ in either condition (not shown for brevity). Labels nodes are significant regions with degree $>4$. Adjacency matrices depicting between-groups differences (PTSD minus control group) in lobe-lobe interactions by hemisphere, for correct recognition trials in the war-related (left panels) and neutral trials (right panels) in the 50-150 $\mathrm{ms}$ time window (dark blue elements indicate $\mathrm{p}>0.05$, warm coloured elements indicate significant differences from permutation testing $\mathrm{p}<$ 0.05). For correct war-related trials, the PTSD showed pronounced, elevated interactions within the right hemisphere, with fronto-temporal, temporo-subcortical and within-subcortical sources displaying elevated connectivity; this pattern was absent for correct neutral trials. 


\section{Discussion}

\section{Summary}

Using working memory and delayed recognition paradigms, we explored memory-related connectivity alterations in soldiers with and without PTSD. Soldiers with PTSD showed comparable working memory performance on a simple 1-back encoding and recognition task compared to a group of combat-exposed control soldiers. Performance was equivalent on a delayed recognition task for war-related images, but the PTSD soldiers exhibited compromised accuracy for recognition of neutral images. In terms of the functional connectivity, both of the tasks revealed elevated, task-dependent alpha synchronisation in the PTSD group around the 50-250 ms time window, particularly within and between fronto-temporal sources, as well as limbic regions, including the hippocampi and amygdalae. Additionally, a decrease in connectivity below baseline levels of node strength for alpha was observed in the delayed recognition task for neutral stimuli in the PTSD group.

A particularly interesting finding was that war-related stimuli in the delayed recognition task recruited additional inter-area interactions in the PTSD group in right frontotemporal connections compared to neutral images, which, along with the behavioural data, suggest an interaction between memory and emotional processing systems mediated by abnormal alpha synchronisation. At the behavioural and neurophysiological levels, the type of repeated image affected recognition differentially between PTSD soldiers and the trauma-resilient control group.

These results indicate that the combat-exposed PTSD group exhibit: 1) alpha hyper synchronisation that underlies several aspects of memory processing, 2) intact working memory performance but compromised delayed recognition for neutral stimuli, 3) recognition of war-related images is associated with increased alpha interactions compared to neutral images, principally within and between right frontotemporal regions, as well as limbic structures, and 4) the magnitude of early whole-brain alpha connectivity during recognition of neutral images was associated with performance accuracy.

\section{Task performance}

Our results demonstrate that soldiers with PTSD were similar in terms of performance to control soldiers in the working memory 1-back task, indexed by reaction time and accuracy. In contrast to our results, it has been reported that the distracting thoughts often prevalent in PTSD, such as traumarelated intrusive memories, hyperarousal/hypervigilance, and impaired and biased attentional control, result in decreased working memory performance [21, 22, 44]. Consistent with Kleim et al. [19], we speculate that the relatively unimpaired performance of our PTSD group is due to the low cognitive demands required for efficient task performance; the 1-back is easy and both groups were operating close to ceiling. With increased attentional and memory load (such as in a 2-back condition), we would predict significantly impaired performance in the PTSD group.
In the delayed recognition task, the PTSD group performed equivalently at discriminating emotionallysalient, war-related stimuli, but were less accurate in the identification of neutral stimuli. We suggest that this is the result of biased attentional allocation, preferentially directed towards trauma-related stimuli. This has been hypothesized to be due to a hyperactive emotional processing network [18, $20,21]$, including the amygdala and ventrolateral and medial prefrontal cortex, and a hypoactive executive control network, including the dorsolateral prefrontal and parietal cortices [18].

\section{MEG connectivity: working memory}

In Experiment 1, the working memory paradigm, we observed robust and elevated, large-scale alpha phase synchrony in the PTSD group, for encoding (from 50-250 $\mathrm{ms}$ ) and immediate recognition (from 150-250 ms) aspects of the paradigm. Alpha synchrony has been heavily implicated in working memory [28, 45-47], with the hypothesised role of this low-frequency rhythmicity purported to be the inter-areal coordination of task-relevant regions to support efficient WM, a process that requires the transient loading, maintenance, and manipulation of pertinent information [9, 10, 27, 48]. It has been shown that the magnitude and the synchrony of alpha oscillations scale with memory load [28, 49], which, given the comparable behavioural performance of the PTSD group in this particular task, suggests elevated alpha acts as a compensatory mechanism in memory processing in PTSD. Previous findings of decreased alpha oscillations in PTSD would appear to contradict these findings [50], but these results were for resting-state voxel wise spectral power, and not inter-area synchronisation examined here. The relation between alterations in evoked responses, source-level spectral power and inter-regional synchronisation would be interesting avenues for future research.

The alpha hyper-connectivity in the PTSD group was observed across a number of regions for encoding and recognition, although seed regions in frontal, temporal and limbic structures were especially synchronised in the left hemisphere for encoding compared to the control group, and right fronto-temporal for recognition. Differential activation patterns in fMRI have been observed for WM paradigms in PTSD, with the amygdala and prefrontal cortices shown to be atypically activated in the disorder [18, 21, 22]. For this particular frequency band and connectivity metric, we found significant increases in PTSD. Phase synchrony is thought to play a functionally-distinct role in mediating communication within neuronal networks compared with amplitude correlation approaches in fMRI studies (for a comprehensive review, see [51]). Previous literature has elucidated the critical role of large-scale alpha synchrony in normal memory function, and the elevated interactions that we found in our PTSD group may be a compensatory mechanism that served to facilitate efficient memory processing.

\section{MEG connectivity: delayed recognition}

In the delayed recognition task correctly identified repeated stimuli induced elevated alpha phase synchrony from 50-150 ms in the combat-related PTSD group compared to a combat-exposed, resilient control group, for both war-related and neutral images. Generally, this suggests that delayed 
recognition of previously viewed stimuli is distinguished by atypical fronto-temporal interactions in PTSD. Specifically, we found that the war-related stimuli were associated with increased interactions within and between sources in the right hemisphere, fronto-temporal cortex and deep-grey matter regions, as might be predicted from fMRI studies on emotional, episodic memory [15], as well as hyper connected seeds in left occipital cortex. Therefore, system-wide network interactions were quantitatively different with the introduction of an emotionally-relevant component to the stimuli, although both aspects of the task were associated with elevated levels of alpha phase synchrony.

Connectivity in the $50-150 \mathrm{~ms}$ time window for alpha strength versus performance positively correlated with individual differences within the groups. Therefore, this suggests that the early alpha synchronisation is critical for performance accuracy within the neutral recognition memory task.

Importantly, we found that correct recognition of emotionally-salient war-related images was associated with increased synchronisation between areas purported to be involved in the emotional memory of episodic events in PTSD, with fMRI studies pointing to the involvement of the amygdalae [52,53] and hippocampi [20,54-56], consistent with other studies proposing a critical role for temporal $[33,34,56]$ and frontal regions $[52,58,59]$. However, these papers report apparently conflicting results, with some finding increased activity, and others decreased activity. These contradictions are likely due in part to the various neuroimaging approaches, as it is not known yet exactly how fMRI activation relates to neurophysiological measures of phase synchronisation, and the functionally-mechanistic interplay between the two is a matter of debate [50]. As well, some of these differences may be due to the use of dissimilar tasks, tapping distinct aspects of memory, which may also account for divergent results.

\section{Conclusions}

To our knowledge, this is the first MEG study of neurophysiological connectivity in PTSD related to memory function. In these analyses we demonstrate that inter-regional alpha-band phase synchronisation, which plays an important role in memory, was elevated differentially in encoding and recognition components of memory function in PTSD during a simple n-back task. During delayed recognition of warrelated stimuli we found that fronto-temporal and deep-grey connections in the right hemisphere were recruited to a greater extent than for the recognition of neutral images in PTSD, suggesting an interaction between emotional and memory systems, mediated by elevated alpha synchrony. These results indicate that alpha synchrony can help explain some of the defining characteristics of PTSD, particularly the biasing of attention towards emotionally-salient stimuli which may be a mechanism mediating the phenomenology of re-experiencing traumatic events often seen in the disorder.

\section{Acknowledgements}

Thank you to Marc Lalancette and Amanda Robertson for help in the data collection, Daniel Cassel for help with data analyses, and Dr. Pang Shek for facilitating this study. This work was supported by funding from Defence Research and Development Canada (DRDC) and the Canadian Forces Health Services (Contract \#: W7719-135182/001/TOR) to MJT and EWP.

\section{Financial Disclosures and Competing Interests}

The authors declare no competing financial interests or potential conflicts of interest.

\section{References}

1. American Psychiatric Association. 2013. Diagnostic and statistical manual of mental disorders (5th ed.). American Psychiatric Publishing, Arlington, VA, USA. doi: 10.1176/appi.books. 9780890425596

2. Kessler RC, Berglund P, Demler O, Jin R, Merikangas KR, et al. 2014. Lifetime prevalence and age-of-onset distributions of DSM-IV disorders in the National Comorbidity Survey Replication. Arch Gen Psychiatry 62(6): 593-602. doi: 10.1001/archpsyc.62.6.593

3. Boulos D, Zamorski MA. 2013. Deployment-related mental disorders among Canadian Forces personnel deployed in support of the mission in Afghanistan, 2001-2008. Canadian Medical Association Journal 185(11): E545-552. doi: 10.1503/cmaj.122120

4. Jenkins MA, Langlais PJ, Delis DA, Cohen RA. 2000. Attentional dysfunction associated with posttraumatic stress disorder among rape survivors. Clin Neuropsychol 14(1): 7-12. doi: 10.1076/13854046(200002)14:1;1-8;FT007

5. Shucard JL, McCabe DC, Szymanski H. 2008. An event-related potential study of attention deficits in posttraumatic stress disorder during auditory and visual $\mathrm{Go} / \mathrm{NoGo}$ continuous performance tasks. Biol Psychol 79(2): 223-233. doi: 10.1016/j.biopsycho.2008.05.005

6. Leskin LP, White PM. 2007. Attentional networks reveal executive function deficits in posttraumatic stress disorder. Neuropsychology 21(3): 275-284. doi: 10.1037/0894-4105.21.3.275

7. Aupperle RL, Melrose AJ, Stein MB, Paulus MP. 2012. Executive function and PTSD: disengaging from trauma. Neuropharmacology 62(2): 686-694. doi: 10.1016/j.neuropharm.2011.02.008

8. Dalgleish T, Taghavi R. 2003. Patterns of processing bias for emotional information across clinical disorders: a comparison of attention, memory, and prospective cognition in children and adolescents with depression, generalized anxiety, and posttraumatic stress disorder. J Clin Child Adolesc Psychol 32(1): 10-21. doi: 10.1207/S15374424JCCP3201_02

9. Samuelson K. 2011. Post-traumatic stress disorder and declarative memory functioning: a review. Dialogues Clin Neurosci 13(3): 346-351.

10. Vasterling JJ, Duke LM, Brailey K, Constans JI, Allain AN, et al. 2002. Attention, learning, and memory performances and intellectual resources in Vietnam veterans: PTSD and no disorder comparisons. Neuropsychology 16(1): 5-14. doi: 10.1037/0894-4105.16.1.5

11. Lindauer RJL, Olff M, van Meijel EPM, Carlier IVE, Gersons BPR. 2006. Cortisol, learning, memory, and attention in relation to smaller hippocampal volume in police officers with posttraumatic stress disorder. Biol Psychiatry 59(2): 171-177. doi: 10.1016/j.biopsych.2005.06.033

12. Wisco BE, Marx BP, Sloan DM, Gorman KR, Kulish AL, et al. 2015. Self-distancing from trauma memories reduces physiological but not subjective emotional reactivity among veterans with posttraumatic stress disorder. Clinical Psychological Science 2167702614560745. doi: $10.1177 / 2167702614560745$ 
13. Steinvorth S, Levine B, Corkin S. 2005. Medial temporal lobe structures are needed to re-experience remote autobiographical memories: evidence from H.M. and W.R. Neuropsychologia 43(4): 479-496. doi: 10.1016/j.neuropsychologia.2005.01.001

14. St-Laurent M, Moscovitch M, Jadd R, McAndrews MP. 2014. The perceptual richness of complex memory episodes is compromised by medial temporal lobe damage. Hippocampus 24(5): 560-576. doi: 10.1002/hipo.22249

15. Addis DR, Moscovitch M, Crawley AP, McAndrews MP. 2004. Recollective qualities modulate hippocampal activation during autobiographical memory retrieval. Hippocampus 14(6): 752-762. doi: 10.1002/hipo.10215

16. McCormick C, St-Laurent M, Ty A, Valiante TA, McAndrews MP. 2013. Functional and effective hippocampal-neocortical connectivity during construction and elaboration of autobiographical aemory retrieval. Cereb Cortex 25(5): 1297-1305. doi: 10.1093/cercor/bht324.

17. Zeithamova D, Preston AR. 2010. Flexible memories: differential roles for medial temporal lobe and prefrontal cortex in crossepisode binding. J Neurosci 30(44): 14676-14684. doi: 10.1523/ JNEUROSCI.3250-10.2010.

18. Hayes JP, Vanelzakker MB, Shin LM. 2012. Emotion and cognition interactions in PTSD: a review of neurocognitive and neuroimaging studies. Front Integr Neurosci 6: 89. doi: 10.3389/fnint.2012.00089

19. Kleim B, Ehring T, Ehlers A. 2012. Perceptual processing advantages for trauma-related visual cues in post-traumatic stress disorder. Psychol Med 42(1): 173-181. doi: 10.1017/S0033291711001048

20. Hayes JP, LaBar KS, McCarthy G, Selgrade E, Nasser J, et al. 2011. Reduced hippocampal and amygdala activity predicts memory distortions for trauma reminders in combat-related PTSD.J Psychiatr Res 45(5): 660-669. doi: 10.1016/j.jpsychires.2010.10.007

21. Dolcos F, McCarthy G. 2006. Brain systems mediating cognitive interference by emotional distraction. J Neurosci 26(7): 2072-2079. doi: 10.1523/JNEUROSCI.5042-05.2006

22. Morey RA, Dolcos F, Petty CM, Cooper DA, Hayes JP, et al. 2009. The role of trauma-related distractors on neural systems for working memory and emotion processing in posttraumatic stress disorder. $J$ Psychiatr Res 43(8): 809-817. doi: 10.1016/j.jpsychires.2008.10.014

23. Tsoory MM, Vouimba RM, Akirav I, Kavushansky A, Avital A, et al. 2008. Amygdala modulation of memory-related processes in the hippocampus: potential relevance to PTSD. Prog Brain Res 167(7): 3551. doi: 10.1016/S0079-6123(07)67003-4

24. Buzsáki G, Wang XJ. 2012. Mechanisms of gamma oscillations. Annu Rev Neurosci 35: 203-225. doi: 10.1146/annurev-neuro-062111-150444

25. Fries P. 2005. A mechanism for cognitive dynamics: neuronal communication through neuronal coherence. Trends Cogn Sci 9(10): 474-480. doi: 10.1016/j.tics.2005.08.011

26. Varela F, Lachaux J. 2001. The brainweb: phase synchronization and large-scale integration. Nature Reviews Neuroscience 2: 229-239. doi: $10.1038 / 35067550$

27. Palva S, Palva JM. 2011. Functional roles of alpha-band phase synchronization in local and large-scale cortical networks. Front Psychol 2: 204. doi: 10.3389/fpsyg.2011.00204

28. Palva JM, Monto S, Kulashekhar S, Palva S. 2010. Neuronal synchrony reveals working memory networks and predicts individual memory capacity. Proc Natl Acad Sci U S A 107(16): 7580-7585. doi: 10.1073/ pnas.0913113107

29. Sauseng P, Klimesch W, Freunberger R, Pecherstorfer T, Hanslmayr S, et al. 2006. Relevance of EEG alpha and theta oscillations during task switching. Exp Brain Res 170(3): 295-301. doi: 10.1007/s00221-0050211-y

30. Sauseng P, Klimesch W, Heise KF, Gruber WR, Holz E, et al. 2009. Brain oscillatory substrates of visual short-term memory capacity. Curr Biol 19(21): 1846-1852. doi: 10.1016/j.cub.2009.08.062

31. Montez T, Poil SS, Jones BF, Manshanden I, Verbunt JPA, et al. 2009.
Altered temporal correlations in parietal alpha and prefrontal theta oscillations in early-stage Alzheimer disease. Proc Natl Acad Sci U S A 106(5): 1614-1619. doi: 10.1073/pnas.0811699106

32. Tewarie P, Schoonheim MM, Stam CJ, van der Meer ML, van Dijk BW, et al. 2013. Cognitive and clinical dysfunction, altered MEG resting-state networks and thalamic atrophy in multiple sclerosis. PloS One 8(7): e69318. doi: 10.1371/journal.pone.0069318

33. Kolassa IT, Wienbruch C, Neuner F, Schauer M, Ruf M, et al. 2007. Altered oscillatory brain dynamics after repeated traumatic stress. $B M C$ Psychiatry 17: 7-56. doi: 10.1186/1471-244X-7-56

34. James LM, Engdahl BE, Leuthold AC, Lewis SM, Van Kampen E, et al. 2013. Neural network modulation by trauma as a marker of resilience: differences between veterans with posttraumatic stress disorder and resilient controls. JAMA Psychiatry 70(4): 410-418. doi: 10.1001/jamapsychiatry.2013.878

35. Dunkley BT, Doesburg SM, Sedge PA, Grodecki RJ, Shek PN, et al. 2014. Resting-state hippocampal connectivity correlates with symptom severity in post-traumatic stress disorder. Neuroimage Clin 5: 377-384. doi: 10.1016/j.nicl.2014.07.017

36. American Psychiatric Association, 2000. Diagnostic and statistical manual of mental disorders (4th ed., text rev.). American Psychiatric Publishing, Washington, DC, USA.

37. Tzourio-Mazoyer N, Landeau B, Papathanassiou D, Crivello F, Etard O, et al. 2002. Automated anatomical labeling of activations in SPM using a macroscopic anatomical parcellation of the MNI MRI singlesubject brain. Neuroimage 15(1): 273-289. doi: 10.1006/nimg.2001.0978

38. Quraan MA, Cheyne D. 2010. Reconstruction of correlated brain activity with adaptive spatial filters in MEG. Neuroimage 49(3): 23872400. doi: 10.1016/j.neuroimage.2009.10.012

39. Muthukumaraswamy SD. 2013. High-frequency brain activity and muscle artifacts in MEG/EEG: a review and recommendations. Front Hum Neurosci 7: 138. doi: 10.3389/fnhum.2013.00138

40. Stam CJ, Nolte G, Daffertshofer A. 2007. Phase lag index: assessment of functional connectivity from multi channel EEG and MEG with diminished bias from common sources. Hum Brain Mapp 28(11): 11781193. doi: $10.1002 / \mathrm{hbm} .20346$

41. Zalesky A, Fornito A, Bullmore ET. 2010. Network-based statistic: identifying differences in brain networks. Neuroimage 53(4): 1197-1207. doi: 1016/j.neuroimage.2010.06.041

42. Zalesky A, Cocchi L, Fornito A, Murray MM, Bullmore E. 2012. Connectivity differences in brain networks. Neuroimage 60(2): 10551062. doi: 10.1016/j.neuroimage.2012.01.068.

43. Xia M, Wang J, He Y. 2013. BrainNet Viewer: a network visualization tool for human brain connectomics. PloS One 8(7): e68910. doi: 10.1371/journal.pone.0068910

44. Schweizer S, Dalgleish T. 2011. Emotional working memory capacity in posttraumatic stress disorder (PTSD). Behav Res Ther 49(8): 498-504. doi: 10.1016/j.brat.2011.05.007

45. Hiltunen T, Kantola J, Abou Elseoud A, Lepola P, Suominen K, et al. 2014. Infra-slow EEG fluctuations are correlated with resting-state network dynamics in fMRI. J Neurosci 34(2): 356-362. doi: 10.1523/ JNEUROSCI.0276-13.2014

46. Palva JM, Palva S, Kaila K. 2005. Phase synchrony among neuronal oscillations in the human cortex. J Neurosci 25(15): 3962-3972. doi: 10.1523/JNEUROSCI.4250-04.2005

47. Palva S,Palva JM.2007. New vistas for alpha-frequency band oscillations. Trends Neurosci 30(4): 150-158. doi: 10.1016/j.tins.2007.02.001

48. Baddeley A. 1992. Working memory. Science 255(5044): 556-559. doi: 10.1126/science. 1736359

49. Jensen O, Gelfand J, Kounios J, Lisman J. 2002. Oscillations in the alpha band $(9-12 \mathrm{~Hz})$ increase with memory load during retention in a short-term memory task. Cereb Cortex 12(8): 877-882. doi: 10.1093/ cercor/12.8.877 
50. Huang MX, Yurgil Ka, Robb A, Angeles A, Diwakar M, et al. 2014. Voxel-wise resting-state MEG source magnitude imaging study reveals neurocircuitry abnormality in active-duty service members and veterans with PTSD. Neuroimage Clin 5: 408-419. doi: 10.1016/j. nicl.2014.08.004

51. Engel AK, Gerloff C, Hilgetag CC, Nolte G. 2013. Intrinsic coupling modes: multiscale interactions in ongoing brain activity. Neuron 80(4): 867-886. doi: 10.1016/j.neuron.2013.09.038

52. Shin L M, Orr S. 2004. Regional cerebral blood flow in the amygdala and medial prefrontalcortex during traumatic imagery in male and female vietnam veterans with PTSD. Arch Gen Psychiatry 61(2): 168176. doi: 10.1001/archpsyc.61.2.168

53. Shin LM, Wright C. 2005. A functional magnetic resonance imaging study of amygdala and medial prefrontal cortex responses to overtly presented fearful faces in posttraumatic stress disorder. Arch Gen Psychiatry 62(3): 273-281. doi: 10.1001/archpsyc.62.3.273

54. Bremner JD, Vythilingam M, Vermetten E, et al. 2003. MRI and PET study of deficits in hippocampal structure and function in women with childhood sexual abuse and posttraumatic stress disorder. $\mathrm{Am} J$
Psychiatry 160(5): 924-932. doi: 10.1176/appi.ajp.160.5.924

55. Etkin A, Wager T. 2007. Functional neuroimaging of anxiety: a metaanalysis of emotional processing in PTSD, social anxiety disorder, and specific phobia. Am J Psychiatry 164(10): 1476-1488. doi: 10.1176/appi. ajp.2007.07030504

56. Shin LM, Liberzon I. 2010. The neurocircuitry of fear, stress, and anxiety disorders. Neuropsychopharmacology 35(1): 169-191. doi: 10.1038/npp.2009.83

57. Engdahl B, Leuthold AC, Tan HRM, Lewis SM, Winskowski AM, et al. 2010. Post-traumatic stress disorder: a right temporal lobe syndrome? J Neural Eng 7(6): 066005. doi: 10.1088/1741-2560/7/6/066005

58. Gold AL, Shin LM, Orr SP, Carson MA, Rauch SL, et al. 2011. Decreased regional cerebral blood flow in medial prefrontal cortex during trauma-unrelated stressful imagery in Vietnam veterans with post-traumatic stress disorder. Psychol Med 41(12): 2563-2572. doi: 10.1017/S0033291711000730

59. Pitman RK, Rasmusson AM, Koenen KC, Shin LM, Orr SP, et al. 2012. Biological studies of post-traumatic stress disorder. Nat Rev Neurosci 13(11): 769-787. doi: 10.1038/nrn3339 